\title{
Energy Current Imaging Method for Time Reversal in Elastic
}

\section{Media}

\author{
Brian E. Anderson \\ Michele Griffa \\ Robert A. Guyer \\ Paul A. Johnson \\ Carene Larmat
}

See next page for additional authors

Follow this and additional works at: https://scholarsarchive.byu.edu/facpub

Part of the Astrophysics and Astronomy Commons, and the Physics Commons

\section{Original Publication Citation}

B. E. Anderson, R. A. Guyer, T. J. Ulrich, P.-Y. Le Bas, C. Larmat, M. Griffa, and P. A. Johnson, "Energy current imaging method for time reversal in elastic media," Appl. Phys. Lett. 95, 2197 (29).

\section{BYU ScholarsArchive Citation}

Anderson, Brian E.; Griffa, Michele; Guyer, Robert A.; Johnson, Paul A.; Larmat, Carene; Le Bas, Pierre-Yves; and Ulrich, Timothy J., "Energy Current Imaging Method for Time Reversal in Elastic Media" (2009).

Faculty Publications. 855.

https://scholarsarchive.byu.edu/facpub/855

This Peer-Reviewed Article is brought to you for free and open access by BYU ScholarsArchive. It has been accepted for inclusion in Faculty Publications by an authorized administrator of BYU ScholarsArchive. For more information, please contact ellen_amatangelo@byu.edu. 


\section{Authors}

Brian E. Anderson, Michele Griffa, Robert A. Guyer, Paul A. Johnson, Carene Larmat, Pierre-Yves Le Bas, and Timothy J. Ulrich 


\title{
Energy current imaging method for time reversal in elastic media
}

\author{
Brian E. Anderson, ${ }^{1,2, a)}$ Robert A. Guyer, ${ }^{1}$ Timothy J. Ulrich, ${ }^{1}$ Pierre-Yves Le Bas, ${ }^{1}$ \\ Carène Larmat, ${ }^{1}$ Michele Griffa, ${ }^{1,3}$ and Paul A. Johnson ${ }^{1}$ \\ ${ }^{1}$ Geophysics Group EES-17, Los Alamos National Laboratory, Los Alamos, New Mexico 87545, USA \\ ${ }^{2}$ Department of Physics and Astronomy, Brigham Young University, Provo, Utah 84602, USA \\ ${ }^{3}$ Laboratory for Building Technologies, Swiss Federal Laboratories for Materials Testing \\ and Research (EMPA), Überlandstrasse 129, Dübendorf 8600, Switzerland
}

(Received 29 January 2009; accepted 25 June 2009; published online 16 July 2009)

\begin{abstract}
An energy current imaging method is presented for use in locating sources of wave energy during the back propagation stage of the time reversal process. During the back propagation phase of an ideal time reversal experiment, wave energy coalesces from all angles of incidence to recreate the source event; after the recreation, wave energy diverges in every direction. An energy current imaging method based on this convergence/divergence behavior has been developed. The energy current imaging method yields a smaller spatial distribution for source reconstruction than is possible with traditional energy imaging methods. () 2009 American Institute of Physics.
\end{abstract}

[DOI: $10.1063 / 1.3180811]$

Time reversal (TR) is a robust method that provides the means to reconstruct and therefore localize a previously unknown source of wave energy both in space and time. ${ }^{1,2} \mathrm{TR}$ is applicable to all types of classical waves, either acoustic, elastodynamic, or electromagnetic. The TR process consists of two phases, the forward propagation and the back propagation. During the forward propagation, the characteristics of the source need not be known; rather one or more detectors record the wave disturbances they experience. These signals are then reversed in time and broadcast from the original detector location(s). The wave energy retraces the original forward propagation paths resulting in a focus of wave energy at the original source location; this constitutes the backward propagation phase when TR focusing occurs.

The backward propagation phase of a TR experiment may be done experimentally or numerically (particularly for problems where the source is internal to a three-dimensional solid and one cannot physically probe the interior). TR has been used in seismology applications to locate earthquake sources. ${ }^{3,4}$ We are currently working on applying a similar imaging method to the one proposed in this letter. TR has also been used in nondestructive evaluation to locate surficial cracks ${ }^{5-7}$ disbonding features just below the sample surface, ${ }^{8}$ and we are currently exploring its use for internal features where the backward propagation is done numerically. Each of these applications, as well as others for fluid media, could benefit from using a higher resolution imaging method, as the one proposed in this work.

An important aspect regarding the TR focusing is the fact that during the back propagation stage of a TR experiment there is an inflow of energy prior to the focal time and an outflow of energy after the focal time, with the focal time defined as the time of maximum energy at the focal location. In order to characterize the energy coalescence that corresponds to the TR focus, one selects a method of processing the wave field data measured during the TR back propagation, defined as the imaging condition. In the following we describe a measurement and processing method that employs

${ }^{\text {a)} E l e c t r o n i c ~ m a i l: ~ b e a @ b y u . e d u . ~}$ the energy current to isolate the focal time and spatial location.

In the experiment, described in detail below, a laser vibrometer is used to measure the out-of-plane particle velocity wave field $v_{z}(\vec{x} ; t)$ as a function of position $\vec{x}=(x ; y)$ on the sample surface, during the backward propagation phase, at each time step of a finite discrete interval about the time of focus. Since information about the displacement field is limited to a single component we form an energy current density vector wave field appropriate to this circumstance

$$
\vec{S}(\vec{x} ; t)=-\tau \frac{\partial u_{z}}{\partial t}(\vec{x} ; t) \vec{\nabla}_{\vec{x}} u_{z}(\vec{x} ; t),
$$

where $v_{z}(\vec{x} ; t)=\partial u_{z} / \partial t(\vec{x} ; t), \quad \vec{\nabla}_{\vec{x}}=(\partial / \partial x ; \partial / \partial y)$, and $\tau$ have units of force per unit length. This equation is analogous to that for the energy current density on a membrane. ${ }^{9}$ In the experiments described below, the Fourier spectrum of the displacement field is centered around $\omega_{0} / 2 \pi=200 \mathrm{kHz}$ so that we may use $u_{z} \approx v_{z} / \omega_{0}$ to write

$$
\vec{S}(\vec{x} ; t)=-\frac{\tau}{\omega_{0}^{2}} \frac{\partial v_{z}}{\partial t}(\vec{x} ; t) \vec{\nabla}_{\vec{x}} v_{z}(\vec{x} ; t)=-k \frac{\partial v_{z}}{\partial t}(\vec{x} ; t) \vec{\nabla}_{\vec{x}} v_{z}(\vec{x} ; t),
$$

where $\tau / \omega_{0}{ }^{2}=k$. We form a scalar measure of the energy flow by considering the flux of $\vec{S}(\vec{x} ; t)$ through a closed rectangular path $\Gamma\left(\vec{x}_{I}\right)$ surrounding a point $\vec{x}_{I}$ on the surface

$$
\Phi\left(\vec{x}_{I} ; t\right)=\oint_{\Gamma\left(\vec{x}_{I}\right)} d \vec{r}(\vec{x}) \cdot \vec{S}(\vec{x} ; t),
$$

where $d \vec{r}(\vec{x})=d l \hat{n}(\vec{x})$ and $\hat{n}(\vec{x})$ is the unit vector normal to the path, pointing outward. The quantity $\Phi$ is called the energy flux. In practical applications this flux field is calculated at each pixel of an image grid. The energy flux, calculated at a specific time, is the imaging condition. Below we compare it with a standard maximum-in-time imaging condition.

To illustrate the merits of the energy current imaging condition, a set of TR experiments is conducted. These experiments utilize a source transducer and a laser vibrometer. The reciprocal TR method, described below, is used to create 
(a)

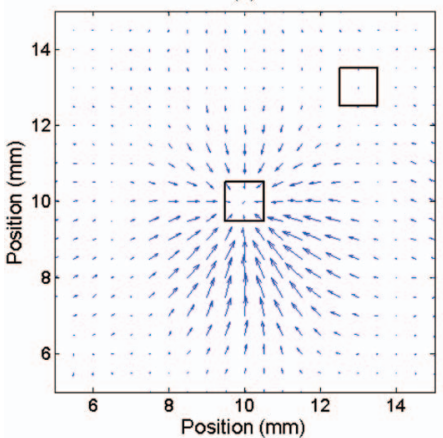

(b)

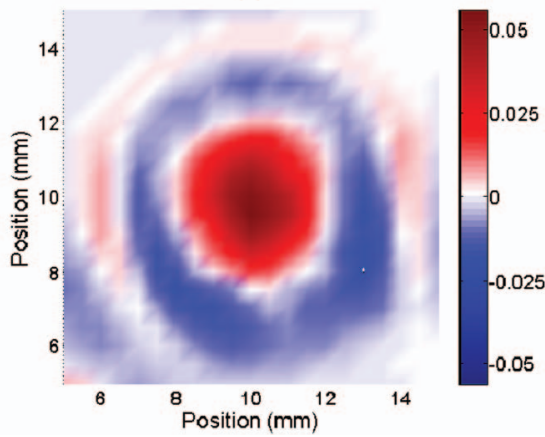

FIG. 1. (Color) Example energy current computation. (a) The instantaneous energy current density vector wave field $\vec{S}$. The length of the arrows indicates the amplitude while the direction of the arrows corresponds to the vector direction. The boxes represent locations where two examples of line integrals are computed, centered at $(10,10) \mathrm{mm}$ (at the source position) and $(13,13) \mathrm{mm}$ (away from the source). (b) The instantaneous energy flux $\Phi\left(\vec{x}_{I} ; t_{\text {pre-focus }}\right)$ just before the focal time. The color bar represents amplitude. pointlike time reversed foci by performing a single channel TR experiment. ${ }^{2,6,8,10-16}$ In the reciprocal TR method, a pulse is emitted from a source transducer and the time dependent response is recorded at a user defined position A. This recorded signal is then reversed in time and broadcast from the original source transducer. Due to spatial reciprocity, a time reversed focus is created at $\mathrm{A},{ }^{13}$ which is akin to creating a virtual source at $A .^{12-16}$ The virtual source method need not be used in every implementation of energy current imaging, rather it is simply a convenient experimental method used for this study. A laser vibrometer is then used to scan a region of space that includes A to determine the quality of the TR spatial focusing.

A glass block of dimensions $89 \times 89 \times 101 \mathrm{~mm}^{3}$ is used to conduct the TR experiments. The glass sample has a shear wave speed $v_{S}=2340 \mathrm{~m} / \mathrm{s}$ and a quality factor $Q=1500$. A lead zirconate titanate disk transducer of $12.7 \mathrm{~mm}$ diameter and $2 \mathrm{~mm}$ thickness is bonded onto the sample with epoxy. The source pulse is a $200 \mathrm{kHz}$ sine wave modulated by a sine squared envelope with a pulse length of $40 \mu$ s, yielding a wavelength of $11.7 \mathrm{~mm}$ corresponding to a center frequency of $200 \mathrm{kHz}$. To enhance the reflectivity of the surface of the sample, reflective tape is placed on it. A rectangular 85 by 85 point scan grid (spacing of $1.0 \mathrm{~mm}$, corresponding to a $1.0 \mathrm{~mm}^{2}$ resolution) for the laser vibrometer is used to image the TR focusing. During the TR back propagation stage of each experiment, the scanning laser vibrometer measures the particle vibration velocity, along one direction, at each point in the scanning grid, $v(\vec{x}, t)$. In the case of these experiments, only the out-of-plane velocity has been measured. However, our TR measurement apparatus at Los Alamos allows us to measure also the in-plane components of the vector velocity wave field, which will be the subject of future research.
Because the wave field measured by the laser vibrometer varies in space, at each time, the total energy flux of Eq. (3) depends on the line chosen to enclose the evaluation point (pixel location of the final image). It is found empirically that the best imaging results are obtained using the eight nearest neighbor scan points about the evaluation point. The $x$ and $y$ spatial derivates at the corner points are each weighted by $1 / \sqrt{2}$.

One common method used for imaging TR foci is the amplitude maximum-in-time imaging condition. For purposes of comparison to the energy current imaging condition, the maximum-in-time imaging condition is squared so that it is proportional to energy

$$
E(\vec{x})=\max _{t \in[0, T]}\left\{\left|v_{z}(\vec{x}, t)\right|^{2}\right\},
$$

where $T$ is the total length of time for the recorded signals.

An example of the current calculation is given in Fig. 1. Figure 1(a) displays the instantaneous energy current density vector wave field, for the TR focusing onto a monopole source, by vector plot; at each point the vector's magnitude and direction are represented by the length and direction of the arrow, respectively. The time at which the snapshot is calculated is just prior to the focal time $t_{\text {pre-focus }}$ when energy is flowing toward the virtual source location. Two boxes are displayed in the plot centered at $(10 ; 10)$ and $(13 ; 13) \mathrm{mm}$, respectively. The box centered at $(10 ; 10) \mathrm{mm}$ is the point of maximum influx of energy, corresponding to the (virtual) source location. The second box shows that the flux of energy is much less away from the focal location (see below). Figure 1(b) displays the instantaneous energy flux calculated from the energy current density vector data displayed in Fig. 1(a), using Eq. (3).

Figure 2 displays the maximum-in-time energy image (a)

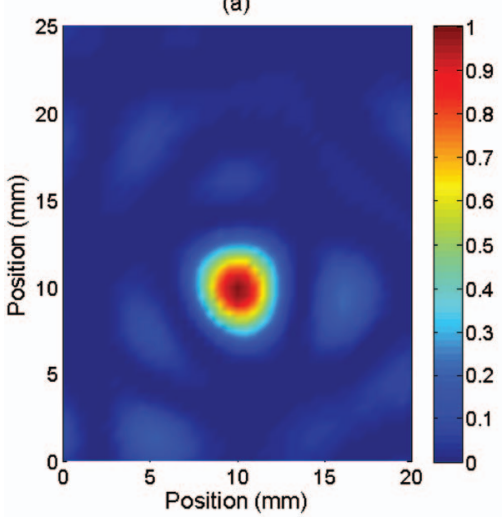

(b)

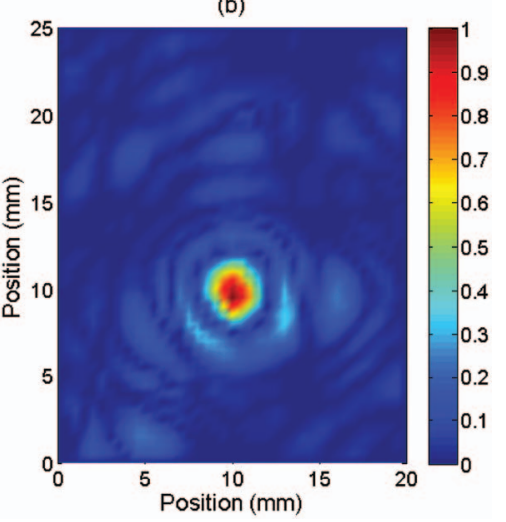

FIG. 2. (Color) Contrasting images of the normalized maximum-in-time energy image (a) and the normalized instantaneous energy current (b) for a single monopole source located at position $(10 ; 10) \mathrm{mm}$ in Fig. 1. Color in these plots represents the magnitude. 


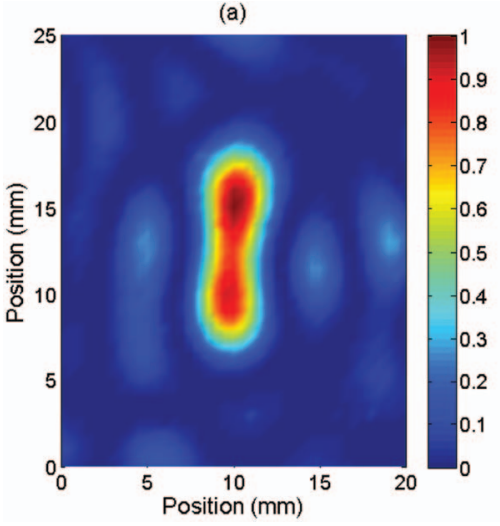

(b)

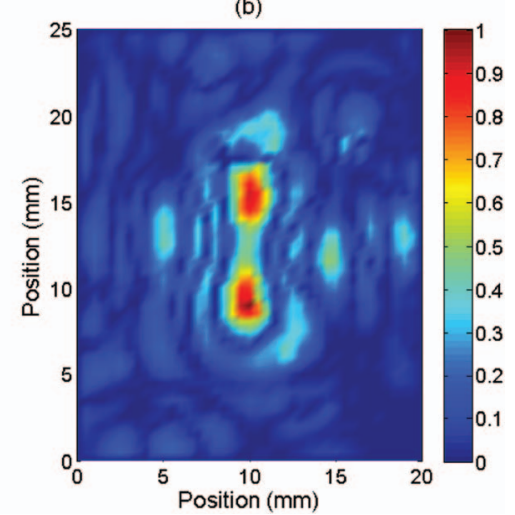

FIG. 3. (Color) Contrasting images of the normalized maximum-in-time energy image (a) and the normalized instantaneous energy current (b) for two coherent monopole sources located at position $(10 ; 10) \mathrm{mm}$ and $(10 ; 16) \mathrm{mm}$. Color in these plots represents the magnitude. from Eq. (4) (a), and the absolute value of the instantaneous energy current just prior to the focal time (b), for a single time reversed focus onto a monopole source, respectively. Two observations can be made regarding the contrasting images, first the spot size in the energy current image is diffraction limited with a $1 / 2$ wavelength diameter, while the focal diameter for the maximum-in-time energy image is $3 / 4$ wavelength (focal spot size is determined from the average null to null distance measured from multiple cross sections of the focal spot). Second the background fringes in the energy current image are slightly higher in amplitude. There is an apparent shift, one pixel down and one to the left, in the peak of Fig. 2(b) with respect to the peak of Fig. 2(a). This shift is likely due to the fact that the flux is greater from that direction than from the other directions, enough to shift it slightly. However, despite this shift in the focal center, one would generally determine the focal location, in a blind experiment, by selecting the center of the two-dimensional focal shape, which in this case is still at the true focal center.

The virtual source experiment can be performed for two different selected locations $\mathrm{A}$ and $\mathrm{B}$, where the forward propagation portions of the experiments are performed separately. The detected signals from each experiment may then be summed and then time reversed. During the backward propagation, the laser vibrometer scan will detect two individual, simultaneously created time reversed foci, which are closely spaced. ${ }^{12}$ In Fig. 3 the maximum-in-time energy image (a), and the absolute value of the instantaneous energy current just prior to the focal time (b), are displayed for two closely spaced foci (spaced $10 \mathrm{~mm}$ or $6 / 7$ wavelength apart), respectively. The energy current image more clearly resolves the two foci than the maximum-in-time energy image.

In summary, when imaging foci during the back propagation phase of a TR experiment, the time dependent energy current imaging condition yields higher resolution imaging of complex sources than does strictly viewing the instantaneous energy. The tradeoff is that the current imaging does result in slightly higher background noise (fringes/speckle). The proposed imaging method should also be applicable to internal sources in solid and fluid media, where the backward propagation portion of the TR experiment is done numerically and the internal wave field in the media is determined.

This work was supported by Institutional Support (LDRD) at the Los Alamos National Laboratory.

${ }^{1}$ M. Fink, Phys. Today 50, 34 (1997).

${ }^{2}$ B. E. Anderson, M. Griffa, C. Larmat, T. J. Ulrich, and P. A. Johnson, Acoust. Today 4, 5 (2008).

${ }^{3}$ C. Larmat, J.-P. Montagner, M. Fink, Y. Capdeville, A. Tourin, and E. Clévédé, Geophys. Res. Lett. 33, L19312 (2006).

${ }^{4}$ C. Larmat, J. Tromp, Q. Liu, and J.-P. Montagner, J. Geophys. Res. 113, B09314, DOI:10.1029/2008JB005607 (2008).

${ }^{5}$ A. M. Sutin, J. A. TenCate, and P. A. Johnson, J. Acoust. Soc. Am. 116, 2779 (2004).

${ }^{6}$ T. J. Ulrich, P. A. Johnson, and A. M. Sutin, J. Acoust. Soc. Am. 119, 1514 (2006).

${ }^{7}$ T. J. Ulrich, P. A. Johnson, and R. A. Guyer, Phys. Rev. Lett. 98, 104301 (2007)

${ }^{8}$ T. J. Ulrich, A. M. Sutin, T. Claytor, P. Papin, P.-Y. Le Bas, and J. A. TenCate, Appl. Phys. Lett. 93, 151914 (2008).

${ }^{9}$ A. L. Fetter and J. D. Walecka, Theoretical Mechanics of Particles and Continua (McGraw-Hill, New York, 1980).

${ }^{10}$ C. Draeger and M. Fink, Phys. Rev. Lett. 79, 407 (1997).

${ }^{11}$ J. de Rosny and M. Fink, Phys. Rev. Lett. 89, 124301 (2002).

${ }^{12}$ M. Scalerandi, A. S. Gliozzi, B. E. Anderson, M. Griffa, P. A. Johnson, and T. J. Ulrich, J. Phys. D 41, 155504 (2008).

${ }^{13}$ T. J. Ulrich, M. Griffa, and B. E. Anderson, J. Appl. Phys. 104, 064912 (2008).

${ }^{14}$ J.-L. Robert and M. Fink, J. Acoust. Soc. Am. 124, 3659 (2008).

${ }^{15}$ B. E. Anderson, T. J. Ulrich, M. Griffa, P.-Y. Le Bas, M. Scalerandi, A. S. Gliozzi, and P. A. Johnson, J. Appl. Phys. 105, 083506 (2009).

${ }^{16}$ B. E. Anderson, R. A. Guyer, T. J. Ulrich, and P. A. Johnson, Appl. Phys. Lett. 94, 111908 (2009). 